\title{
Quantification of hip effusion-synovitis and its cross-sectional and longitudinal associations with hip pain, MRI findings and early radiographic hip $O A$
}

\author{
Harbeer Ahedi ${ }^{1,2,3^{*}}$, Dawn Aitken ${ }^{1}$, Leigh Blizzard ${ }^{1}$, Flavia Cicuttini ${ }^{4}$ and Graeme Jones ${ }^{1}$
}

\begin{abstract}
Background: Hip effusion-synovitis may be relevant to osteoarthritis (OA) but is of uncertain etiology. The aim of this study was to describe the cross-sectional and longitudinal associations of hip effusion-synovitis with clinical and structural risk factors of $O A$ in older adults.

Methods: One hundred ninety-six subjects from the Tasmanian Older Adult Cohort (TASOAC) study with a right hip STIR (Short T1 Inversion Recovery) Magnetic Resonance Imaging (MRI) on two occasions were included. Hip effusion-synovitis CSA $\left(\mathrm{cm}^{2}\right)$ was assessed quantitatively. Hip pain was determined by WOMAC (Western Ontario and McMaster Universities Osteoarthritis) while hip bone marrow lesions (BMLs), cartilage defects (femoral and/or acetabular) and high cartilage signal were assessed on MRI. Joint space narrowing (0-3) and osteophytes (0-3) were measured on x-ray using Altman's atlas.

Results: Of 196 subjects, 32\% ( $n=63)$ had no or a small hip effusion-synovitis while 68\% $(n=133)$ subjects had a moderate or large hip effusion-synovitis. Both groups were similar but those with moderate or large hip effusionsynovitis were older, had higher BMI and more hip pain. Cross-sectionally, hip effusion-synovitis at multiple sites was associated with presence of hip pain [Prevalence ratio (PR):1.42 95\%Cl:1.05,1.93], but not with severity of hip pain. Furthermore, hip effusion-synovitis size associated with femoral defect (ßeta:0.32 95\%Cl:0.08,0.56). Longitudinally, and incident hip cartilage defect (PR: 2.23 95\%Cl:1.00, 4.97) were associated with an increase in hip effusion-synovitis CSA. Furthermore, independent of presence of effusion-synovitis, hip BMLs predicted incident (PR: 1.62 95\%Cl: 1.13, 2.34) and worsening of hip cartilage defects (PR: 1.50 95\%Cl: 1.20, 1.86). While hip cartilage defect predicted incident (PR: 1.11 95\%Cl: 1.03, 1.20) and worsening hip BMLs (PR: 1.16 95\%Cl: 1.04, 1.30).

Conclusions: Hip effusion-synovitis at multiple sites (presumably reflecting extent) may be associated with hip pain. Hip BMLs and hip cartilage defects are co-dependent and predict worsening hip effusion-synovitis, indicating causal pathways between defects, BMLs and effusion-synovitis.
\end{abstract}

Keywords: Musculoskeletal disorders, Osteoarthritis, Hip pain, Effusion-synovitis, Articular cartilage, Subchondral BMLs, Radiological hip OA

\footnotetext{
* Correspondence: Harbeer.ahedi@utas.edu.au

${ }^{1}$ Menzies Institute for Medical Research, University of Tasmania, Hobart, Tasmania, Australia

${ }^{2}$ Institute of Musculoskeletal Health, Sydney, NSW, Australia

Full list of author information is available at the end of the article
}

C The Author(s). 2020 Open Access This article is licensed under a Creative Commons Attribution 4.0 International License, which permits use, sharing, adaptation, distribution and reproduction in any medium or format, as long as you give appropriate credit to the original author(s) and the source, provide a link to the Creative Commons licence, and indicate if changes were made. The images or other third party material in this article are included in the article's Creative Commons licence, unless indicated otherwise in a credit line to the material. If material is not included in the article's Creative Commons licence and your intended use is not permitted by statutory regulation or exceeds the permitted use, you will need to obtain permission directly from the copyright holder. To view a copy of this licence, visit http://creativecommons.org/licenses/by/4.0/. The Creative Commons Public Domain Dedication waiver (http://creativecommons.org/publicdomain/zero/1.0/) applies to the data made available in this article, unless otherwise stated in a credit line to the data. 


\section{Background}

Osteoarthritis (OA) is characterized by alterations in composition, structure and function of the various components of joint, including synovium [1-4]. OA has been historically categorized as non-inflammatory arthritis [2, 4]. However, synovitis plays a key role in cartilage damage and vice versa $[1,2,5]$. Occurring in either early or late stages of OA, synovitis leads to increase in catabolic and proinflammatory mediators such as cytokines, nitric oxide, prostaglandin E, and neuropeptides. These mediators produce excess proteolytic enzymes, which cause cartilage matrix degradation. In turn, cartilage breakdown leads to worsening synovitis $[2,6]$.

Several studies have reported associations of knee effusion-synovitis proving that it is one of the causes of knee pain, has an adverse effect on cartilage and is linked with radiographic knee OA [3, 7-10]. Although effusion-synovitis is a significant clinical prognostic factor for OA [11], at the hip it remains under-investigated.

A small retrospective study was the first to report hip effusion in twelve out of twelve subjects and severe synovitis in nine out of twelve subjects with hip RDOA (Rapidly Destructive OA) but did not demonstrate correlations of hip effusion or synovitis [12]. Subsequently, in a clinical study, hip effusion was reported in $70 \%$ of the subjects and major or/and asymmetrical hip effusion associated not only with hip pain but also with hip radiographic OA (ROA) [13]. In a study, Magnetic Resonance Imaging (MRI) was used for evaluating hip effusion and synovitis separately, a weak association between grade 1 synovitis (but not grade 2) and hip pain was found. However, in subjects with either synovitis or effusion, severe hip ROA was prevalent [14]. In a retrospective study, extensive synovitis was found in subjects with RDOA than in those with hip OA, indicating that higher synovitis could be related with rapid disease progression [15]. Modest correlations between hip effusion and hip ROA were demonstrated in a study designed to evaluate hip OA. However, no association between hip effusionsynovitis and hip pain score [16]. Interestingly, an MRI study conducted in athletics demonstrated lower prevalence of effusion-synovitis in hips with pain than without pain (Odds Ratio: 0.46 (95\% CI: 0.3, 0.8) [17].

In a small study, investigating the effect of hyaluronic acid (HA) in injection in those with and without hip OA reported that hip pain was correlated with effusionsynovitis $(r=0.27, p=0.03)$ and was lower in participants injected with HA (13.9 vs 7.8, $P<0.001)$ [18] Another study, showed that the frequency of effusion $(p=0.013)$ and reactive synovitis $(p<0.001)$ was greater in those with cam impingement [19].

Overall, the etiology of hip effusion-synovitis is underreported, and current data is inconclusive. Due to its proximity to the hip cartilage and other structures, hip effusion-synovitis could be a significant progenitor of hip OA. Effusion-synovitis could be a potential target for future clinical trials [20]. Hence, this study aims to describe the cross-sectional and longitudinal associations of hip effusion-synovitis in a large community-based sample.

\section{Methods \\ Subjects}

The Tasmanian Older Adult Cohort (TASOAC) study is an ongoing prospective, population-based study initiated in 2002 and has been extensively described in previous studies [21]. During the TASOAC study, a hip protocol was added during the latter part of phase 2. Hip MRI scans for phase 2 and phase 3 were conducted approximately 2.3 years apart. In the current study a sample of 245 consecutive participants who had a STIR (Short T1 Inversion Recovery) MRI sequence at phase 2 and/or phase 3 were included. Of these 245 participants, 30 participants were lost to follow-up in phase 3 and 17 subjects had no STIR MRI at phase 2. Of 198 subjects, hip effusion-synovitis could not be adequately assessed in the MRI scans of 2 subjects and these were excluded. Accordingly, a total of 196 subjects with complete data were included in this study. Written informed consent was obtained from all participants and the Southern Tasmanian Health and Medical Human Research Ethics Committee approved this study (approval number: H6488).

\section{Clinical and hip pain measures}

Height, weight, Body mass index (BMI) were measured using standard protocols. Hip pain was determined using a hip specific Western Ontario and McMaster Universities osteoarthritis (WOMAC) index pain score. WOMAC uses a ten-point scale from 0 (indicating no pain) to 9 (indicating severe pain). Hip pain (five items) was assessed using the following questions: 'Referring to your hips only, how much pain did you experience when walking on flat surface, going up and down the stairs, at night while in bed, sitting or lying, and standing upright.' These five items were summed to create a total hip pain score, each with a possible range from 0 to 45 [22].

\section{Magnetic resonance imaging (MRI) assessment}

For those with a hip MRI, the right hip was imaged in the sagittal plane using a 1.5 Telsa G. E signal wholebody magnetic resonance unit with a phased-array flex coil. Two MRI sequences were conducted for each participant. Sagittal images were obtained at a partition thickness of $1.5 \mathrm{~mm}$ with an in-plane resolution of $0.39 \times 0.39 \mathrm{~mm}(512 \times 512$ pixels $)$ using, a T1-weighted, fat-suppressed, 3-dimensional gradient-recalled acquisition in the steady state. The parameters for this were: 
flip angle 55 degrees; repetition time $58 \mathrm{~ms}$; echo time 12 $\mathrm{ms}$; inversion time (IT) $130 \mathrm{~ms}$; field of view $16 \mathrm{~cm}$; 60 partitions; $512 \times 512$-pixel matrix; acquisition time 11 mins $56 \mathrm{~s}$, and one acquisition [23]. A second set of sagittal images was obtained with a slice thickness of $3.5 \mathrm{~mm}$ and an inter-slice gap of $1.5 \mathrm{~mm}$ using a STIR-weighted, fat saturation two-dimensional fast spin echo sequence. This sequence used a repetition time $4340 \mathrm{~ms}$, echo time $28.4 \mathrm{~ms}$; field of view $20 \mathrm{~cm} ; 15$ partitions (16 slices) and $512 \times 512$ pixel matrix [24]. All MRI measures were conducted on STIR MRI sequence using OsiriX imaging software (University of Geneva, Geneva, Switzerland).

\section{Quantitative assessment of hip effusion-synovitis}

For quantitative measurements of hip effusion-synovitis, the observer (HA) selected the MRI slice with the largest effusion-synovitis and measured the maximum crosssectional area (CSA) by drawing contours around the outer edges (Fig. 1). If the effusion-synovitis was present at more than one site around the femoral head (anterior, posterior or both), then the largest CSA of effusionsynovitis on each site was assessed. The reproducibility was evaluated in 40 subjects, with a 4 weeks interval between the two measures. The intra-rater agreement (kappa) for the presence of hip effusion-synovitis was 0.84, and the intra-class correlation coefficient (ICC) for hip effusion-synovitis CSA was 0.97 . We have used similar method to assess knee hip effusion-synovitis [8] and also used these measurements in previously published studies $[25,26]$. For analyses, the population was divided into two groups by median of effusion-synovitis CSA. The first group included subjects with no or small $\left(<0.77 \mathrm{~cm}^{2}\right)$ hip effusion-synovitis and the second group included subjects with moderate or large hip effusion-synovitis $\left(\geq 0.77 \mathrm{~cm}^{2}\right)$.

\section{Assessment of hip cartilage defects, hip BMLs, and high cartilage signal}

Hip cartilage defects were assessed on MRI using OsiriX (Fig. 2). Hip defects on either femoral head or acetabulum were identified as any change in the hip cartilage and were categorized as; grade $0=$ normal cartilage, grade $1=$ focal blistering or irregularities on the cartilage surface or a partial thickness defect and grade 2 = full-thickness defect with bone ulceration and/ or exposure of bone. If more than one defect was present at one site, the highest score was used. In a reliability study of 40 subjects with re-measurements after 4 weeks, the intra-rater agreement (kappa) was 0.89 . Furthermore, the inter-rater reliability (kappa) assessed by two readers $(n=40)$ for the presence of cartilage defects and defect categories was 0.84 and 0.63 respectively [25].

Hip BMLs were identified as areas of increased signal intensity adjacent to the subchondral bone on the femoral head and/or the acetabulum. The observer manually selected the MRI slice with the largest BML and then determined the BML size $\left(\mathrm{cm}^{2}\right)$ (Fig. 2). Intra-observer repeatability was assessed and the intra-class correlation coefficient (ICC) of the hip, femoral and acetabular BMLs was 0.98, 0.96 and 0.99 respectively [24].

High cartilage signal intensity change $[27,28]$ was defined as a high signal intensity band within the hip cartilage either adjacent to a hip BML or at any location on the STIR MRI slice if there was no BML present (Fig. 2). The intra-rater agreement (kappa) was 0.88 [24].

Methods for assessing hip cartilage defects, hip BMLs and cartilage were adapted by previously published grading systems used for assessment of structural changes in the knee detected by MRI [21, 29-32].

\section{Hip radiographs}

Antero-posterior weight-bearing radiographs of the pelvis were obtained. Hip $\mathrm{x}$-rays were read by two trained readers using the OARSI (Osteoarthritis Research Society international) grading system. The radiographic features of JSN and osteophytes of the right hip were graded on a 4-point scale, ranging from 0 to 3 where $0=$ no disease and $3=$ most severe disease by using an Altman's atlas [33]. The intra-observer reliability for $\mathrm{x}$ -

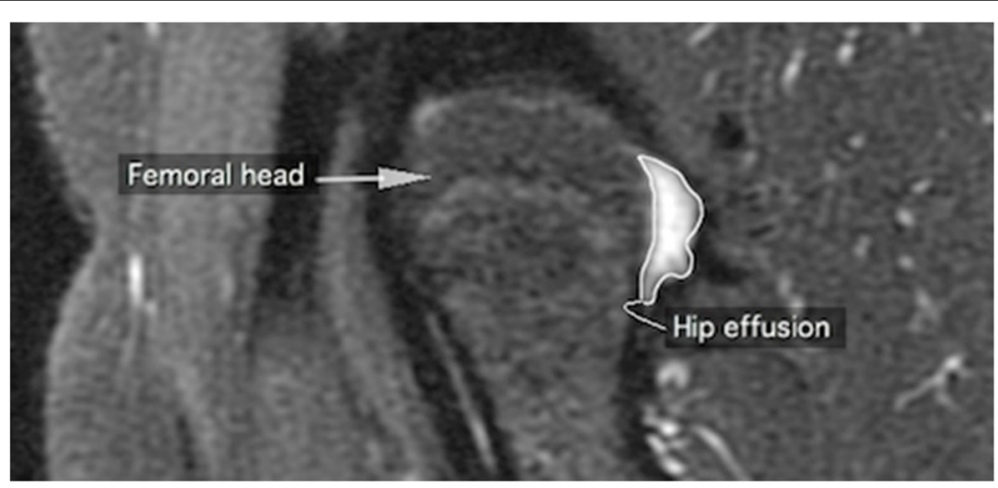

Fig. 1 Assessment of hip effusion-synovitis using OsiriX imaging software 

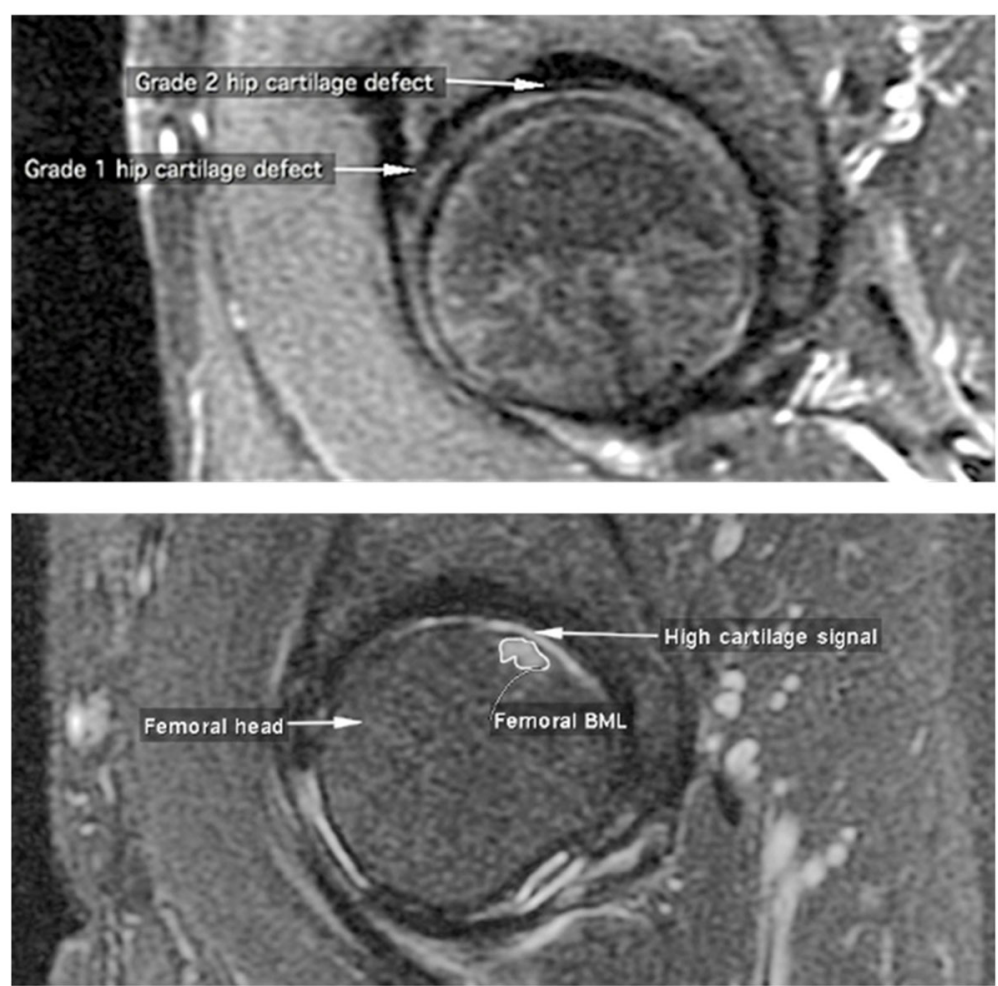

Fig. 2 Measurement of hip BML, high cartilage signal and hip cartilage defects using OsiriX imaging software

rays was carried out in 40 subjects and the ICC scores ranged from $0.60-0.87$ [23, 34]. A non-zero score of either JSN or osteophytes was regarded as evidence of hip ROA. Thus, after combining JSN and osteophytes score, the presence of hip ROA was defined as a total score of 1 or greater.

\section{Statistical analysis}

Ninety-four percent of the population had any hip effusion-synovitis. We were unable to differentiate between physiological (e.g. normal joint fluid) and pathological effusion-synovitis. Initial data-driven cut-off points did not reveal any significant results.,

Differences in demographical characteristics between participants who had no or small and moderate or large hip effusion-synovitis was calculated by using unpaired t-tests and chi-square tests (Table 1). Hip effusionsynovitis was also analyzed by the number of sites affected (independent of size) and continuously as CSA.

For cross-sectional analysis, log-binomial regression was employed to estimate the association between presence of hip pain, moderate/large hip effusion-synovitis and presence of hip effusion-synovitis at one or two/three sites. For estimating the relationship between severity of hip pain and categories of hip effusion-synovitis, linear regression of the logarithm of pain score on a binary covariate for hip effusion-synovitis was used. Log-binominal regression models were applied to investigate the associations between presence of hip BMLs, high cartilage signal, hip cartilage defects, hip ROA and presence of hip effusion-synovitis while linear regression was used to test the relationship between these factors and hip effusionsynovitis CSA. Associations between presence of hip BMLs and presence of cartilage defects were also investigated using similar models.

For longitudinal analyses, linear regression models were administered to estimate the relationship between change in hip pain and change in hip effusion-synovitis CSA. Similarly, the association between change in prevalence of hip BMLs, hip cartilage defects, hip ROA (baseline only) and change in hip effusion-synovitis CSA was examined using linear regression. Log-binominal regression was employed to investigate if hip BMLs predicted incident and worsening of cartilage defects and viceversa (from phase 2 to phase 3 ). All results were presented as prevalence ratios (PR) and the models were adjusted for age, sex, body mass index (BMI), hip BMLs and hip cartilage defects as required. For cross-sectional analysis only, data on subjects at phase 2 and phase 3 were combined in analyses, and the correlation between repeated measurements on individuals was taken into account by adjusting standard errors using the sandwich (robust) estimator of variance [35, 36]. All statistical tests were two-sided and $p$ values $<0.05$ were considered 
Table 1 Characteristics of the sample population

\begin{tabular}{|c|c|c|c|}
\hline Characteristics & Small or no hip effusion-synovitis $(N=63)$ & Moderate or large hip effusion-synovitis $(N=133)$ & $P$-value \\
\hline Age (yrs): mean (SD) & $63.4(4.88)$ & $64.6(4.97)$ & 0.04 \\
\hline Male sex (\%) & $46 \%(29 / 63)$ & $44 \%(58 / 133)$ & 0.75 \\
\hline BMI $\left(\mathrm{kg} / \mathrm{cm}^{2}\right)$ : mean (SD) & $27.2(3.11)$ & $28.0(3.10)$ & 0.02 \\
\hline \multicolumn{4}{|l|}{ Hip pain } \\
\hline Presence & $33 \%(19 / 63)$ & $44 \%(58 / 133)$ & 0.04 \\
\hline Severity: mean (SD) & $1.82(5.41)$ & $1.81(5.45)$ & 0.98 \\
\hline High cartilage signal & $52 \%(33 / 63)$ & $59 \%(77 / 133)$ & 0.20 \\
\hline Presence of any bone marrow lesions (BMLs) & $21 \%(13 / 63)$ & $15 \%(20 / 133)$ & 0.12 \\
\hline \multicolumn{4}{|l|}{ Hip cartilage defects } \\
\hline Femoral defects & $54 \%(34 / 63)$ & $57 \%(75 / 133)$ & 0.69 \\
\hline Acetabular defects & $68 \%(42 / 63)$ & $65 \%(86 / 133)$ & 0.61 \\
\hline Any hip defects & $71 \%(44 / 63)$ & $71 \%(94 / 133)$ & 0.92 \\
\hline Presence of radiographic hip OA (ROA) & $44 \%(27 / 63)$ & $50 \%(66 / 133)$ & 0.59 \\
\hline
\end{tabular}

The variable small or no hip effusion-synovitis includes participants with hip effusion-synovitis less than $0.77 \mathrm{~cm}^{2}$ and the variable moderate/large hip effusion includes participants with effusion-synovitis more than equal to $0.77 \mathrm{~cm}^{2}$ Data presented as means (SD) and proportions

Bold indicates statistically significant results $(p<0.05)$

significant and were conducted using Intercooled Stata 12 for Mac (Stata Corp, College station, TX, USA).

\section{Results}

Overall, 196 participants from both phases were included in these analyses and the characteristics of the population is presented in Table 1. The population was split into two groups by the median of effusion-synovitis size. Participants with moderate/large effusion were older, heavier and mostly males. Presence of hip pain was more common in those with moderate/large hip effusion-synovitis, than in those with small or no hip effusion-synovitis. However, no differences were found in pain severity (hip pain $>0$ ). For the imaging markers, of the two groups $20 \%$ of participants with small or no hip effusion-synovitis had a hip BML. Furthermore, presence of high cartilage signal and radiological hip OA was proportionate in both groups. Although those with moderate or large effusion-synovitis had more defects, no statistically significant differences were found.

Table 2 shows the cross-sectional associations between the presence and severity of hip pain and categories of hip effusion-synovitis. Overall, subjects with moderate/ large hip effusion-synovitis had 31\% greater hip pain but this association was not statistically significant. Nevertheless, those with hip effusion-synovitis at multiple sites had $42 \%$ higher hip pain in comparison to those with hip effusion- synovitis at only one site. Hip effusionsynovitis did not associate with severity of hip pain.

Cross-sectionally, hip BMLs [(Prevalence Ratio (PR): 0.75 95\%CI:0.36,1.60)], high cartilage signal (PR:1.01 95\%CI:0.85,1.21), hip cartilage defects (PR:1.12 95\%CI: 0.88,1.42) and hip ROA (PR:0.94 95\%CI: 0.74,1.20) were not associated with the presence of hip effusionsynovitis. Nevertheless, any hip BMLs associated with any hip cartilage defects (PR: 1.22 95\%CI 1.06, 1.40) independent of presence of hip effusion-synovitis. For these analyses; prevalence ratios (95\% confidence intervals) were adjusted for age, sex, body mass index, presence of hip BMLs, presence of hip cartilage defects as required and clustering of observations on subjects at phase 2 and phase 3 was used using Huber-White estimator of variance.

Table 2 Cross sectional associations between presence of hip pain and categories of hip effusion-synovitis

\begin{tabular}{|c|c|c|}
\hline Study factor & Presence of hip pain & Severity of hip pain \\
\hline Categories of hip effusion-synovitis & Adjusted PR $(95 \% \mathrm{Cl})^{\mathrm{a}}$ & Ratio of means $(95 \% \mathrm{Cl})^{\mathrm{b}}$ \\
\hline Presence of moderate/large hip effusion-synovitis & $1.31(0.98,1.74)$ & $0.81(0.53,1.08)$ \\
\hline Presence of hip effusion-synovitis at two/three sites & $1.42(1.05,1.93)$ & $0.99(0.70,1.40)$ \\
\hline
\end{tabular}

Independent variable: presence and severity of hip pain. Dependent variable: hip effusion-synovitis (moderate/large \& multiple sites)

The variable small or no hip effusion-synovitis includes participants with hip effusion-synovitis less than $0.77 \mathrm{~cm}^{2}$ and the variable moderate/large hip effusion includes participants with effusion-synovitis more than equal to $0.77 \mathrm{~cm}^{2}$

${ }^{\text {a PR }}(95 \% \mathrm{Cl})=$ prevalence ratios $(95 \%$ confidence intervals) adjusted for age, sex, BMI, presence of hip BMLs, presence of cartilage defects and with clustering of observation on subjects at phase 2 and phase 3 taken into account

${ }^{\mathrm{b}}$ Ratio of means (95\% confidence intervals) adjusted for age, sex, BMI, presence of hip BMLs, presence of hip cartilage defects and with clustering of observation on subjects at phase 2 and phase 3 taken into account 
Independent of presence of hip BMLs, hip effusionsynovitis associated with femoral cartilage defects (ßeta: $0.3295 \% \mathrm{CI} 0.08,0.56$ ). No other structural or radiographic features of hip showed statistically significant associations.

Table 3 summarizes the longitudinal association between change in hip pain and change in hip effusionsynovitis CSA. Although, resolving hip effusion-synovitis showed a reduction in hip pain and worsening or persistent hip effusion- synovitis showed an increase in hip pain these analyses were not statistically significant.

Table 4 outlines the longitudinal associations between change in effusion-synovitis, any hip BMLs and hip cartilage defects. Change in effusion-synovitis was not associated with BMLs but increased the risk of incident of hip cartilage defect by two-folds.

Further longitudinal analyses demonstrated that any hip BML predicted incident (PR: 1.62 95\%CI: 1.13, 2.34) and worsening hip cartilage defects (PR: 1.50 95\%CI: $1.20,1.86)$. Conversely, any hip cartilage defect predicted incident (PR: 1.11 95\%CI: 1.03, 1.20) and worsening hip BMLs (PR: 1.16 95\%CI: 1.04, 1.30). These analyses were independent of presence of hip effusion-synovitis.

\section{Discussion}

This prospective cohort study describes the correlates of hip effusion-synovitis. Overall, there was no association between hip effusion-synovitis and hip pain. However, presence of hip effusion-synovitis at multiple sites (presumably reflecting effusion-synovitis extent) was associated with the presence of hip pain. Femoral defects were associated with hip effusion-synovitis CSA. High cartilage signal did not associate with hip effusion-synovitis and contradictory to previous research no relationship between hip ROA and hip effusion-synovitis was found. Change in effusion-synovitis increased the risk of hip cartilage defects.

Cross-sectionally, subjects with a hip effusion- synovitis at multiple sites were $42 \%$ more likely to have hip pain. At the hip, a few studies besides ours have reported these associations. The first study demonstrated that older adults with diagnosed hip OA with hip pain in mid-thigh and pain on palpation had higher odds of major hip effusion-synovitis [13]. While in the second study, a weak association was found between hip pain and grade1 but not grade2 synovitis [14]. The third found no correlation between hip effusion-synovitis and hip pain score [16]. Oddly, the fourth study showed higher prevalence of effusion-synovitis in athletics with lower hip pain [17] and the last study reported hip pain and effusion-synovitis were corelated.

These studies lack longitudinal data and measured effusion or synovitis semi-quantitatively. Also, only one of the above studies measured synovitis and effusion separately. While our study has its strengths, we did not assess site-specific hip pain and a cross-sectional association was found only in subjects who had extensive hip effusion-synovitis. We could not differentiate between physiological or pathological joint fluid but overall, it appears that site and extent of effusionsynovitis at the hip may be relevant for pain in OA.

Cross-sectionally, hip effusion-synovitis size was associated with femoral cartilage defects. Subsequently, in the longitudinal analyses, change in hip effusion-synovitis predicted incident of hip cartilage defects. An association between hip cartilage defects and hip effusion-synovitis has not been previously reported but most of the existing evidence for knee OA coincides with our findings. For instance, subjects with large knee effusion(>grade 2) at baseline had 2.7 times greater risk of cartilage damage at the end of 30 months follow-up [3]. A recent longitudinal study reported that regional knee effusion-synovitis predicted knee cartilage defects; cartilage volume loss and knee BMLs and these associations were largely mediated by cartilage defects [8]. Hence, causal pathways exist between knee effusion and knee cartilage defects; and suggests that knee cartilage defects could lead to the development of BMLs and cartilage volume loss at the knee joint [8]. Our results are comparable with these findings. Since there are limited number of studies on hip

Table 3 Longitudinal associations between change in presence of hip effusion-synovitis and change in hip pain from baseline to follow up

\begin{tabular}{llll}
\hline Study factor & $\mathrm{n}$ & Change in hip pain & $\begin{array}{l}\text { Change in hip pain } \\
\text { Further adjusted } \beta \text { eta }(95 \% \mathrm{Cl})^{\mathrm{b}}\end{array}$ \\
\hline No hip effusion-synovitis & 8 & Ref & Ref \\
Resolved hip effusion-synovitis & 11 & $-0.20(-1.51,1.20)$ & $-0.31(-1.82,1.20)$ \\
Worsening or persistent hip effusion-synovitis & 174 & $+0.32(-0.81,1.46)$ & $+0.30(-0.91,1.52)$ \\
\hline
\end{tabular}

Independent variable: change in hip pain. Dependent variable: change in hip effusion-synovitis CSA/size

$\mathrm{n}=$ number of observations in the analyses

Bold indicates statistically significant results

CSA cross-sectional area

${ }^{a}$ Beta co-efficient (95\% confidence intervals) adjusted for age, sex and body mass index

${ }^{\mathrm{b}}$ Beta co-efficient (95\% confidence intervals) further adjusted for hip BMLs and hip cartilage defects at phase2 
Table 4 Longitudinal associations between change in hip effusion-synovitis, any hip BMLs and cartilage defects

\begin{tabular}{|c|c|c|c|c|c|}
\hline \multirow[t]{3}{*}{ Study factor } & \multicolumn{3}{|l|}{ Any hip BMLs } & \multicolumn{2}{|l|}{ Hip Cartilage defects } \\
\hline & Resolved & Incident & Persistent & Incident & Persistent \\
\hline & Adjusted PR $(95 \% \mathrm{Cl})$ & Adjusted PR (95\%Cl) & Adjusted PR $(95 \% \mathrm{Cl})$ & Adjusted PR (95\%Cl) & Adjusted PR $(95 \% \mathrm{Cl})$ \\
\hline \multicolumn{6}{|c|}{ Change in hip effusion-synovitis CSA } \\
\hline Model 1 & $0.65(0.30,1.43)$ & $1.31(0.62,2.75)$ & $0.94(0.46,1.91)$ & $2.23(1.01,4.91)$ & $0.99(0.88,1.12)$ \\
\hline Model 2 & $0.67(0.32,1.39)$ & $1.25(0.66,2.33)$ & $0.94(0.49,1.80)$ & $2.23(1.00,4.97)$ & $1.00(0.89,1.33)$ \\
\hline
\end{tabular}

Independent variable: presence of structural/ radiographic factors. Dependent variable: change in hip effusion-synovitis size PR $(95 \% \mathrm{Cl})=$ prevalence ratios $(95 \%$ confidence intervals)

Mode1 1 = prevalence ratios ( $95 \%$ confidence intervals) adjusted for age, sex, body mass index at phase 2

Mode1 2 = prevalence ratios ( $95 \%$ confidence intervals) adjusted for age, sex, body mass index, presence of hip BMLs, presence of cartilage defects at phase 2

Bold indicates statistically significant results

CSA cross-sectional area

effusion-synovitis the nature of this study is mostly exploratory and further research is warranted.

Hip BMLs did not associate with hip effusionsynovitis. Nevertheless, independent of hip effusionsynovitis hip BMLs predicted worsening and incident of hip cartilage defects, and vice versa. Increase in hip effusion-synovitis increased the risk of developing cartilage defects.

We speculate that catabolic and proinflammatory mediators triggered by synovitis lead to intra-articular debris due to cartilage breakdown. In turn, presence of articular debris causes further inflammation of the synovium [1, 2, 4, 11]. Hypothetically, an increase in joint intra-capsular pressure could push synovitis into the subchondral bone through the cartilage defects causing the formation of BMLs. Moreover, BMLs are known to correlate with not only knee subchondral bone mineral density (BMD) [37] but also with local hip BMD [38, 39]. Thus, suggesting a causal pathway between hip effusion-synovitis, hip defects, hip BMLs and alterations in the bone itself $[38,39]$. Moreover, effusion-synovitis is associated with hip shape [40] and studies show that those with cam impingement are more likely to have effusion-synovitis [19, 41].

\section{Limitations}

We assessed hip effusion-synovitis quantitatively and have used similar methods previously $[24,42]$ and obtained high reproducibility. Nevertheless, the STIR MRI sequence used to examine joint effusion did not allow separation of physiological and pathological effusion and contrast-enhanced technique may yield clearer results. But our findings match with other MRI-based reports using similar techniques $[13,16]$. Longitudinal analyses were carried out in a small number of subjects with hip BMLs. However, our findings were statistically significant and coincided with existing literature.

\section{Conclusions}

Hip effusion-synovitis at multiple sites (presumably reflecting extent) may be associated with hip pain. Hip
BMLs and hip cartilage defects are co-dependent and predict worsening hip effusion-synovitis, indicating causal pathways between defects, BMLs and effusionsynovitis.

Together, these factors have a deleterious effect on the bone and also contribute towards progression of OA.

\section{Abbreviations}

BML: Bone marrow Lesions; BMI: Body Mass Index; ICC: Intraclass correlation coefficient; Cl: Confidence interval; CSA: Cross sectional area;

FAl: Femoroacetabular impingement; IT: Inversion time; KL: KellgrenLawrence; JSN: Joint space narrowing; OA: Osteoarthritis; MRI: Magnetic Resonance Imaging; OARSI: Osteoarthritis Research Society International; PR: Prevalence Ratios; RDOA: Rapidly destructive Osteoarthritis; ROA: Radiological Osteoarthritis; STIR: Short-TI Inversion Recovery; TASOAC: Tasmanian Older Adult cohort study; WOMAC: Western Ontario and McMaster Universities Osteoarthritis Index

\section{Acknowledgements}

A special thanks go to the participants of the TASOAC study. We thank Catrina Boon, Pip Boon and Dr. Ming Lu for their contributions. We would also like to extend our gratitude towards Dr. David Connell, M. D for providing his expertise and guidance.

\section{Authors' contributions}

$H A, D A, F C$, and GJ contributed to the conception and design of the study. HA, LB and GJ contributed in analyses of the data. DC and GJ provided HA with training and guidance for assessment of hip effusion-synovitis. HA performed hip effusion-synovitis measurements and prepared the first draft of the manuscript. All authors contributed in data interpretation, critical revision and final approval of the manuscript. HA assumes responsibility for the integrity and accuracy of the data.

\author{
Authors' information \\ 1,2 Harbeer Ahedi, PhD. \\ ${ }^{1}$ Dawn Aitken (PhD, Post-doctoral research fellow) is supported by an NHMR \\ C Early Career Fellowship. \\ ${ }^{1}$ Leigh Blizzard (PhD, Associate Professor) is supported by an NHMRC Career \\ Development Fellowship. \\ ${ }^{1}$ Graeme Jones (FRACP, MD, Professor) is supported by an NHMRC \\ Practitioner Fellowship.

\section{Funding} \\ The TASOAC study was supported by the National Health and Medical \\ Research Council of Australia, Tasmanian Community Fund, Masonic \\ Centenary Medical Research Foundation, Royal Hobart Hospital Research \\ Foundation and Arthritis Foundation of Australia.
}

\section{Availability of data and materials}

The datasets used and/or analyzed for the current study are available from the corresponding author. 


\section{Ethics approval and consent to participate}

Written informed consent was obtained from all participants and the Southern Tasmanian Health and Medical Human Research Ethics Committee approved this study (approval number: H6488).

\section{Consent for publication}

Not applicable.

\section{Competing interests}

The authors have no conflict of interest.

\section{Author details}

${ }^{1}$ Menzies Institute for Medical Research, University of Tasmania, Hobart, Tasmania, Australia. ${ }^{2}$ Institute of Musculoskeletal Health, Sydney, NSW, Australia. ${ }^{3}$ Sydney School of Public Health, The University of Sydney Faculty of Medicine and Health, Sydney, New South Wales, Australia. ${ }^{4}$ Department of Epidemiology and Preventive Medicine, School of Public Health and Preventive Medicine, Monash University, Alfred Hospital, Melbourne, VIC 3004, Australia.

Received: 5 December 2019 Accepted: 23 July 2020

Published online: 10 August 2020

\section{References}

1. Scanzello C, Goldring S. The role of synovitis in osteoarthritis pathogenesis. Bone. 2012;51(2):249-57.

2. Sellam J, Berenbaum F. The role of synovitis in pathophysiology and clinical symptoms of osteoarthritis. Nat Rev Rheumatol. 2010;6(11):625-35.

3. Roemer F, Guermazi A, Felson D, Niu J, Nevitt M, Crema M, Lynch J, Lewis C, Torner J, Zhang Y. Presence of MRI-detected joint effusion and synovitis increases the risk of cartilage loss in knees without osteoarthritis at 30month follow-up: the MOST study. Ann Rheum Dis. 2011;70(10):1804-9.

4. Mathiessen A, Conaghan PG. Synovitis in osteoarthritis: current understanding with therapeutic implications. Arthritis Res Ther. 2017;19(1):18.

5. Mobasheri A, Saarakkala S, Finnilä M, Karsdal MA, Bay-Jensen A-C, van Spil WE. Recent advances in understanding the phenotypes of osteoarthritis. F1000Res. 2019;8:F1000 Faculty Rev-2091.

6. Grässel S, Muschter D. Recent advances in the treatment of osteoarthritis. F1000Res. 2020;9:F1000 Faculty Rev-1325.

7. Hill CL, Hunter DJ, Niu J, Clancy M, Guermazi A, Genant H, Gale D, Grainger A, Conaghan P. Synovitis detected on magnetic resonance imaging and its relation to pain and cartilage loss in knee osteoarthritis. Ann Rheum Dis. 2007:66(12):1599-603.

8. Wang X, Blizzard L, Halliday A, Han W, Jin X, Cicuttini F, Jones G, Ding C. Association between MRI-detected knee joint regional effusion-synovitis and structural changes in older adults: a cohort study. Ann Rheum Dis. 2014;75(3):519-25

9. Baker K, Grainger A, Niu J, Clancy M, Guermazi A, Crema M, Hughes L, Buckwalter J, Wooley A, Nevitt M, et al. Relation of synovitis to knee pain using contrast-enhanced MRIs. Ann Rheum Dis. 2010;69(10):1779-83.

10. Ishijima M, Watari T, Naito K, Kaneko H, Futami I, Yoshimura-Ishida K, Tomonaga A, Yamaguchi H, Yamamoto T, Nagaoka I, et al. Relationships between biomarkers of cartilage, bone, synovial metabolism and knee pain provide insights into the origins of pain in early knee osteoarthritis. Arthritis Res Ther. 2011;13(1):R22.

11. Burke CJ, Alizai H, Beltran LS, Regatte RR. MRI of synovitis and joint fluid. J Magn Reson Imaging. 2019;49(6):1512-27.

12. Boutry N, Christelle P, Xavier L, Fredoux D, Migaud H, Cotten A. Rapidly destructive osteoarthritis of the hip: MR imaging findings. Am J Roentgenol. 2002;179:657-63.

13. Bierma-Zeinstra S, Bohnen A, Verhaar J, Prins A, Ginai-Karamat A, Lameris J. Sonography for hip joint effusion in adults with hip pain. Ann Rheum Dis. 2000;59(3):178-82.

14. Roemer F, Hunter D, Winterstein A, Li L, Kim Y, Cibere J, Mamisch T, Guermazi A. Hip osteoarthritis MRI scoring system (HOAMS): reliability and associations with radiographic and clinical findings. Osteoarthr Cartil. 2011 19(8):946-62.

15. Birn J, Pruente R, Avram R, Eyler W, Mahan M, van Holsbeeck M. Sonographic evaluation of hip joint effusion in osteoarthritis with correlation to radiographic findings. J Clin Ultrasound. 2014;42(4):205-11.
16. Lee S, Nardo L, Kumar D, Wyatt C, Souza R, Lynch J, McCulloch C, Majumdar S, Lane N, Link T. Scoring hip osteoarthritis with MRI (SHOMRI): a whole joint osteoarthritis evaluation system. J Magn Reson Imaging. 2015;41(6): 1549-57.

17. Heerey J, Srinivasan R, Smith A, Kemp J, Pizzari T, King M, Lawrenson P, Scholes M, Souza R, Majumdar S. Prevalence of osteoarthritis features on MRI in high-impact athletes: the femoroacetabular impingement and hip osteoarthritis cohort study. Osteoarthr Cartil. 2020;28:S263.

18. Deseyne N, Conrozier T, Lellouche H, Maillet B, Weber U, Jaremko JL, Paschke J, Epstein J, Maksymowych WP, Loeuille D. Hip inflammation MRI scoring system (HIMRISS) to predict response to hyaluronic acid injection in hip osteoarthritis. Joint Bone Spine. 2018;85(4):475-80.

19. Anbar A, Ragab Y, Zeinhom F, El-Shaarawy N, Emad Y, Abo-Elyoun I, Hussein $\mathrm{H}$, Rasker JJ. Cam versus pincer femoroacetabular impingement. Which type is associated with more hip structural damage? An exploratory crosssectional study. Current Orthopaedic Practice. 2017;28(2):188-94.

20. Haraden CA, Huebner JL, Hsueh M-F, Li Y-J, Kraus VB. Synovial fluid biomarkers associated with osteoarthritis severity reflect macrophage and neutrophil related inflammation. Arthritis Res Ther. 2019;21(1):146.

21. Dore D, Quinn S, Ding C, Winzenberg T, Zhai G, Cicuttini F, Jones G. Natural history and clinical significance of MRI detected bone marrow lesions at the knee: a prospective study in community dwelling older adults. Arthritis Res Ther. 2010;12(6):R223.

22. Bellamy B, Buchanan W, Goldsmith C, Camplbell J, Stitt L. Validation study of WOMAC: a health status instrument for measuring clinically important patient relevant outcomes to antirheumatic drug therapy in patients with osteoarthritis of the hip or knee. J Rheumatol. 1988;15(12):1833-40.

23. Zhai G, Cicuttini F, Srikanth V, Cooley H, Ding C, Jones G. Factors associated with hip cartilage volume measured by magnetic resonance imaging: the Tasmanian older adult cohort study. Arthritis Rheum. 2005:52(4):1069-76.

24. Ahedi H, Aitken D, Blizzard L, Cicuttini F, Jones G. A population-based study of the association between hip bone marrow lesions, high cartilage signal, and hip and knee pain. J Clin Rheumatol. 2013;33(3):369-76.

25. Ahedi H, Aitken DA, Blizzard LC, Ding CH, Cicuttini FM, Jones G. Correlates of hip cartilage defects: a cross-sectional study in older adults. J Rheumatol. 2016:43(7):1406-12.

26. Mezhov V, Laslett LL, Ahedi H, Blizzard CL, Aspden RM, Gregory JS, Saunders FR, Graves S, Lorimer M, Munogoda IP, et al. Predictors of total hip replacement in community based older adults: a cohort study. Osteoarthr Cartil. 2019;27:S79-80

27. Totterman S, Tamez-Pena J, Schreyer E, Gonzalez P, Hunter DJ. Cartilagebone contrast behavior in OAl progression sub-cohort: correlation to WOMAC scores. Osteoarthr Cartil. 2009;17(Suppl 1):S74-5.

28. King L, Higgs J, Aisen A, Buckwalter K, Martel W, McCune J. MRI in osteoarthritis of the hip: Gradiations of severity. Magn Reson Imaging. 1988;6:229-36.

29. Dore D, Martens A, Quinn S, Ding C, Winzenberg T, Zhai G, Pelletier JP, Martel-Pelletier J, Abram F, Cicuttini F, et al. Bone marrow lesions predict site-specific cartilage defect development and volume loss: a prospective study in older adults. Arthritis Res Ther. 2010;12:R222.

30. Ding C, Garnero P, Cicuttini F, Scott F, Cooley H, Jones G. Knee cartilage defects: association with early radiographic osteoarthritis, decreased cartilage volume, increased joint surface area and type II collagen breakdown. Osteoarthr Cartil. 2005:13(3):198-205.

31. Carnes J, Stannus O, Cicuttini F, Ding C, Jones G. Knee cartilage defects in a sample of older adults: natural history, clinical significance and factors influencing change over 2.9 years. Osteoarthr Cartil. 2012;20(12):1541-7.

32. Stannus $\mathrm{O}$, Jones $\mathrm{G}$, Cicuttini F, Ding C. Cartilage signal intensity on MRl association with body mass index, cartilage defects and type II collagen breakdown. Osteoarthr Cartil. 2011;19(Suppl. 1):S191-2.

33. Altman RD, Hochberg M, Murphy W, Wolfe F, Lequesne M. Atlas of individual radiographic features in osteoarthritis. Osteoarthr Cartil. 1995; 3(Suppl):3-70

34. Ding C, Cicuttini F, Boon C, Boon P, Srikanth V, Cooley H, Jones G. Knee and hip radiographic osteoarthritis predict total hip bone loss in older adults: a prospective study. J Bone Miner Res. 2010;25:858-65.

35. Huber $P$. The behavior of maximum likelihood estimates under nonstandard conditions. In: Proceedings of the fifth Berkeley symposium on mathematical statistics and probability: 1967. Berkeley: Univerisity of California Press; 1967. p. 221-3.

36. White $\mathrm{H}$. A Heteroskedasticity-consistent covariance matrix estimator and a direct test for Heteroskedasticity. Econometrica. 1980;48(4):817-38. 
37. Dore D, Quinn S, Ding C, Winzenberg T, Jones G. Correlates of subchondral BMD: a cross-sectional study. J Bone Miner Res. 2009;24(12):2007-15.

38. Ahedi H, Aitken D, Blizzard L, Cicuttini F, Jones $G$. The association between hip bone marrow lesions and bone mineral density: a cross-sectional and longitudinal population-based study. Osteoarthr Cartil. 2013;19(13):838.

39. Lo GH, McAlindon TE, Niu J, Zhang Y, Beals C, Dabrowski C, Le Graverand MP, Hunter DJ. Bone marrow lesions and joint effusion are strongly and independently associated with weight-bearing pain in knee osteoarthritis: data from the osteoarthritis initiative. Osteoarthr Cartil. 2009;17(12):1562-9.

40. Ahedi H, Aspden R, Blizzard L, Saunders F, Cicuttini F, Aitken D, Jones G, Gregory J. Hip shape as a predictor of osteoarthritis progression in a prospective population cohort. Arthritis Care Res. 2017;69(10):1566-73.

41. Ahedi H, Winzenberg T, Bierma-Zeinstra S, Blizzard L, Van Middelkoop M, Waarsing J, Cicuttini F, Jones G. Does Femoroacetabular impingement (FAl) correlate with measures of hip OA? Osteoarthr Cartil. 2017:25:S333-4.

42. Dore D, Martens A, Quinn S, Ding C, Winzenberg T, Zhai G, Pelletier J, Martel-Pelletier J, Abram F, Cicuttini F, et al. Bone marrow lesions predit sitespecific cartilage defect development and volume loss: a prospective study in older adults. Arthritis Res Ther. 2010;12(6):R222.

\section{Publisher's Note}

Springer Nature remains neutral with regard to jurisdictional claims in published maps and institutional affiliations.

Ready to submit your research? Choose BMC and benefit from:

- fast, convenient online submission

- thorough peer review by experienced researchers in your field

- rapid publication on acceptance

- support for research data, including large and complex data types

- gold Open Access which fosters wider collaboration and increased citations

- maximum visibility for your research: over $100 \mathrm{M}$ website views per year

At $\mathrm{BMC}$, research is always in progress.

Learn more biomedcentral.com/submissions 